\title{
The Importance of DNA Repair in Tumor Suppression
}

\author{
Yisroel Brumer and Eugene I. Shakhnovich \\ Harvard University, 12 Oxford Street, Cambridge, Massachusetts 02138
}

(Dated: October 25, 2018)

\begin{abstract}
The transition from a normal to cancerous cell requires a number of highly specific mutations that affect cell cycle regulation, apoptosis, differentiation, and many other cell functions. One hallmark of cancerous genomes is genomic instability, with mutation rates far greater than those of normal cells. In microsatellite instability (MIN tumors), these are often caused by damage to mismatch repair genes, allowing further mutation of the genome and tumor progression. These mutation rates may lie near the error catastrophe found in the quasispecies model of adaptive RNA genomes, suggesting that further increasing mutation rates will destroy cancerous genomes. However, recent results have demonstrated that DNA genomes exhibit an error threshold at mutation rates far lower than their conservative counterparts. Furthermore, while the maximum viable mutation rate in conservative systems increases indefinitely with increasing master sequence fitness, the semiconservative threshold plateaus at a relatively low value. This implies a paradox, wherein inaccessible mutation rates are found in viable tumor cells. In this paper, we address this paradox, demonstrating an isomorphism between the conservatively replicating (RNA) quasispecies model and the semiconservative (DNA) model with post-methylation DNA repair mechanisms impaired. Thus, as DNA repair becomes inactivated, the maximum viable mutation rate increases smoothly to that of a conservatively replicating system on a transformed landscape, with an upper bound that is dependent on replication rates. On a specific single fitness peak landscape, the repair-free semiconservative system is shown to mimic a conservative system exactly. We postulate that inactivation of post-methylation repair mechanisms are fundamental to the progression of a tumor cell and hence these mechanisms act as a method for prevention and destruction of cancerous genomes.
\end{abstract}

PACS numbers: 87.14Gg, 87.17.-d, 87.23.-n

\section{INTRODUCTION}

Cancer has presented itself as one of the most difficult challenges science has ever faced. The complexity of the disease, experimental obstacles, and the vast array of tumor types have made characterization of the many facets of tumor progression a slow process. It is now understood that this progression requires the alteration of numerous genes, as a genome progresses from its normal state to a full-blown cancer cell $1^{\underline{1}}$. One important aspect of the cancerous genome lies in its genetic instability. All cancerous genomes display either high mutation rates (in MIN tumors) or chromosomal instability (in CIN tumors) ${ }^{2}$.

One of the most successful theoretical methods for studying genomic evolution at high mutation rates has been Eigen's quasispecies mode $\underline{l}^{3}$. This model considers an explicit population of genomes, each made up of $L$ nucleotides chosen from an alphabet of size $S$, usually chosen to be two for simplicity or four to model the nucleotides in nature. These genomes replicate, mutate and compete on a chosen fitness landscape, a unique mapping of genotype to fitness. This is often accomplished by assigning different replication rates to each possible genome and setting all death rates to be equal. The model has yielded a number of impressive and experimentally verified predictions ${ }^{4,5,6.7}$ and has recently been used as the basis for novel anti-viral therapies.8. The main prediction lies in the idea of an error catastrophe. Below a threshold mutation rate, dubbed the "error threshold", the population evolves, independent of starting conditions, to a distribution of genomes near the sequence of maximal fitness, often called the master sequence. Above the threshold mutation rate or the "error threshold", the population reaches a random distribution with no discernible master. This crossover is depicted in Fig. 1. These ideas provide a method for destroying RNA-based viral genomes. Viruses are expected to evolve a mutation rate slightly below the error threshold so as to maintain the capacity to rapidly adapt without surpassing the error threshold and becoming inviable. Hence, by increasing the mutation rate of the species, the virus can be destroyed, and this technique has been successfully applied ${ }^{4}$.

These ideas have recently been suggested to apply to cancer cells ${ }^{9}$. Cancer and RNA viruses share genetic instability in the sense that both are rapidly mutating and recent work has focused on the idea that mutagens may push cancer cells past the error threshold in a similar manner. Support for the idea that the quasispecies model can be applied to complex cellular genomes comes from recent studies that yielded accurate qualitative and quantitative predictions for complex systems such as the adaptive immune system ${ }^{5}$.

However, past work on the quasispecies model has focused on conservatively replicating systems such as RNA 10.11 .12 .13 .14 .15 . In these systems, single stranded genomes are copied to produce a new, possibly error prone, strand without affecting the original. In semiconservative systems like DNA, double stranded genomes unzip to produce two single strands, each of which is copied to produce a new complementary strand by WatsonCrick base-pairing. A variety of mismatch repair enzymes 


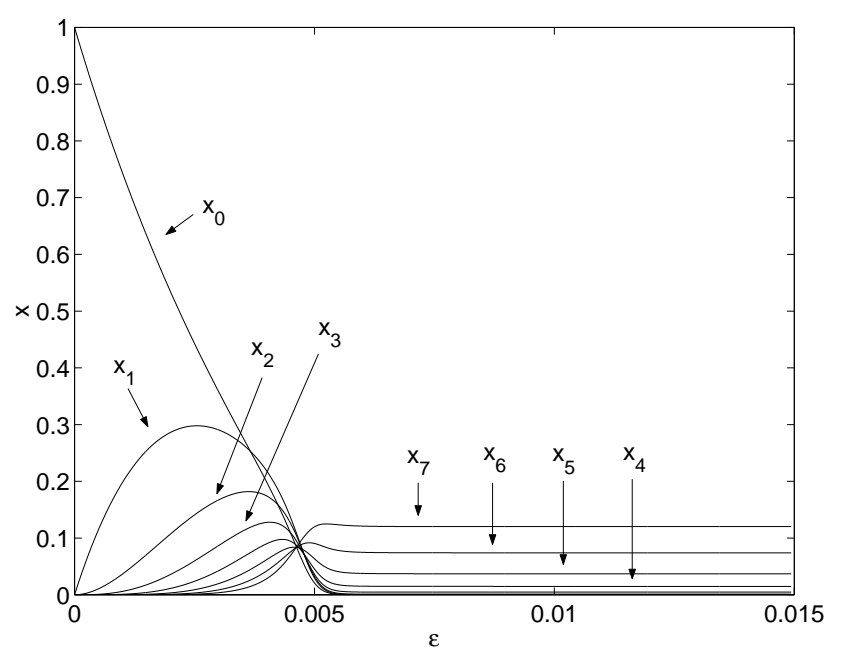

FIG. 1: A schematic diagram illustrating the error catastrophe predicted by the quasispecies model. The concentration of sequences of Hamming distance $i$ from the master sequence is represented by $x_{i}$ and plotted against the mutation rate, $\epsilon$. At low concentrations, the master sequence (of Hamming distance 0), dominates the population, but is surrounded by a cloud of closely related genomes . Above the error threshold, this clustering disappears, and we see a random distribution of genomes, where each Hamming class has a concentration proportional to its size.

then repair any errors in the new strand, keeping the effective error rate low. A few errors remain, though, and these, as well as extrinsic mutations induced by UV radiation or other mutagens, are repaired by post-methylation repair enzymes that cannot distinguish between the new and old strands. Thus, some of these base pair mismatches are repaired in the old strand and the original strand is not conserved ${ }^{16}$ as shown schematically in Fig. 2.

The quasispecies model has recently been extended to incorporate this behavior ${ }^{17}$. It was found that, contrary to popular assumption, the semiconservative system displays fundamentally different behavior than the conservative system. In particular, on a single fitness peak landscape, the semiconservative error catastrophe occurs at significantly lower mutation rates than the conservative case $\frac{17}{\underline{1}}$. Particularly interesting is that, in the conservative case, the maximum viable mutation rate increases without bounds with increasing master sequence replication rate, while the semiconservative system reaches a threshold value $\frac{17}{\underline{1}}$, and this has been confirmed by simulation for finite populations sizes and genome lengths. Thus, for the conservative case, it is always possible to "out-replicate" the error threshold. That is, for any given mutation rate, there exists a relative fitness for the master sequence such that, if the master sequence has that or greater fitness, the error catastrophe is avoided. This is not true for semiconservative genomes, where there exist mutation rates that cause the error catastrophe for (a)

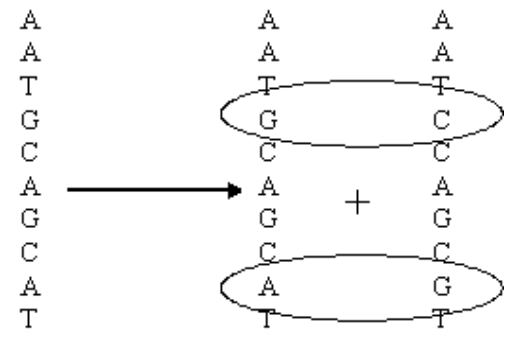

(b)

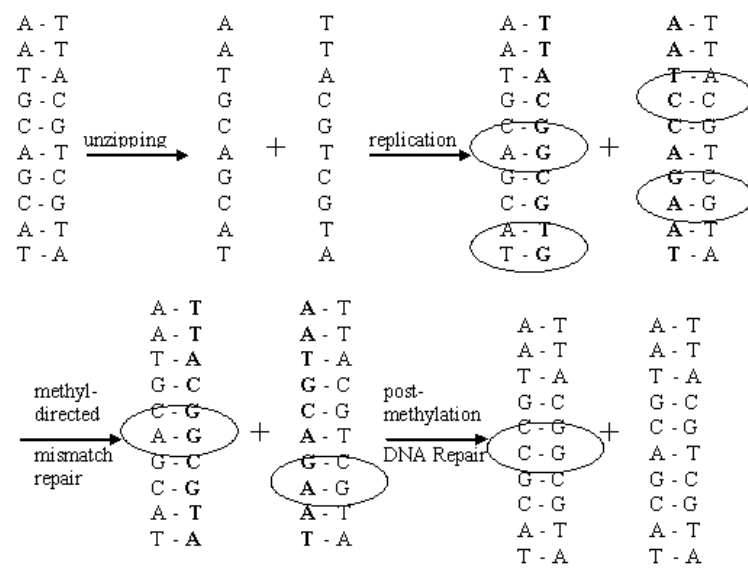

FIG. 2: A schematic model of (a) conservative and (b) semiconservative replication. Non-methylated strands are bolded and errors are circled.

any value of the master sequence fitness. We note in passing that a conservative system will also follow this behavior if the possibility of unrepaired extrinsic mutation is incorporated. The fundamentally different nature of the semiconservative error catastrophe has numerous implications ${ }^{7}$, but is particularly pertinent to the study of cancer.

Within the conservative paradigm, it is reasonable to assume that cancer cells are capable of maintaining a viable population of rapidly mutating genomes, as rapid replication rates are one of the hallmarks of cancerous cells 1 . However, the recent results on the semiconservative system present a paradox. The mutation rates in MIN cancer cells, known to be 50-1000 times higher than those of normal cells $\frac{18.19}{}$, certainly lie higher than any reasonable value for the low semiconservative threshold (for example, the single fitness peak landscape yields 1.39 errors/genome/replication as an upper bound for the error threshold in a long semiconservative genome, while cancer cells display error rates over three orders of magnitude greater). Furthermore, the rapid replication rates that allow such high mutation rates in the conservative case provide no help, as the maximum allowed mutation rate cannot exceed a rather low threshold value, no matter how fast the cancer cells replicate. Hence, these 
recent results appear to present a paradox: rapidly mutating genomes are prevalent in cancerous cells, but such high mutation rates should exceed the error threshold and hence yield inviable genomes.

In this paper, we address this paradox and demonstrate that a semiconservative system can mimic a conservative population through the degradation of postmethylation lesion repair. In section II we discuss the isomorphism between conservative replication and semiconservative replication without lesion repair. In section III we look at the implications of this result and in section IV we present our conclusions.

\section{DNA REPAIR AND SEMICONSERVATIVE REPLICATION}

As discussed above, DNA replication can be considered a three part process; unzipping, complementary strand creation and mismatch repair. Afterwards, any remaining mismatches, as well as damage caused by environmental conditions, are repaired by a set of repair enzymes. Global genomic repair (GGR) fixes lesions, errors and mismatches along the entire genome, while transcription coupled repair (TCR) subjects the expressed portion of the genome to more careful scrutiny and repair.

In appendix $\mathrm{A}$, we use the quasispecies equations to demonstrate a mathematical isomorphism between a population of conservatively replicating genomes and semiconservatively replicating genomes without any lesion repair. In this case, the semiconservative system behaves, in essence, like a conservatively replicating system on a transformed landscape. Each single stranded genome produces one, possibly error prone, complementary copy. Although mismatch repair may keep the effective error rate low, the lack of lesion repair ensures that the original strand is unaffected by these errors. Hence, each genome replicates, in essence, conservatively, but with the added wrinkle that each single stranded genome remains attached to the strand that either created it or that it most recently created, yielding a system that replicates conservatively on a transformed fitness landscape.

To make this more rigorous, appendix B presents the full solution to the semiconservative quasispecies evolving on a specific single fitness peak landscape. This is plotted in Fig. 3, along with the conservative and semiconservative solutions to the same problem. While the semiconservative error threshold clearly plateaus at high $\sigma$, the repair-free semiconservative case mimics a conservative system, as the error threshold increases indefinitely with increasing $\sigma$.

Lastly, it is important to consider the case where lesion repair is partially active, as complete degradation of lesion repair is not likely to occur in nature. In appendix $\mathrm{C}$, the single fitness peak quasispecies is reconsidered, this time with partially active lesion repair. The error threshold is shown to increase smoothly from the semiconservative to the conservative threshold as shown

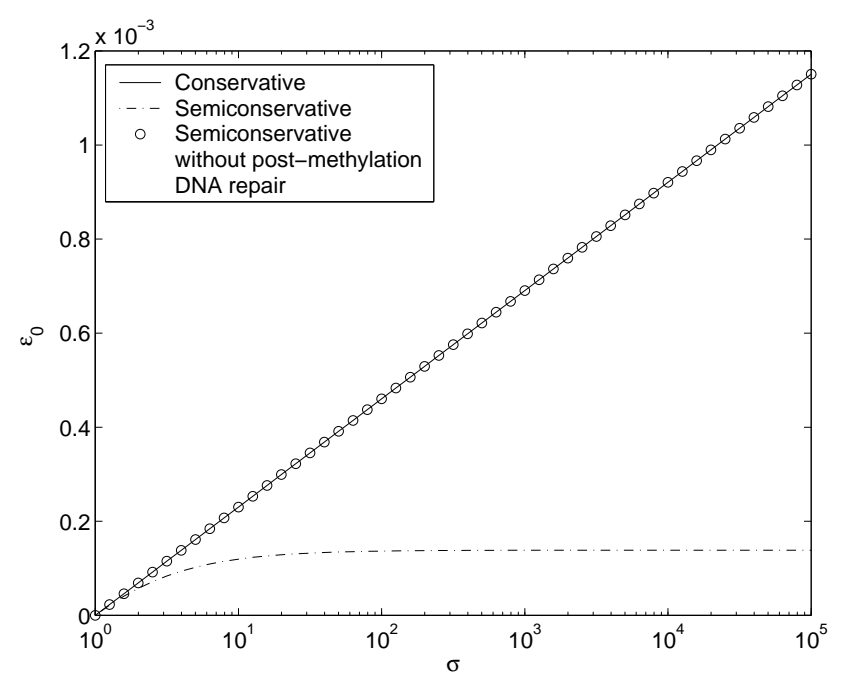

FIG. 3: The value of the error threshold $\left(\epsilon_{0}\right)$ vs. the fitness of the master sequence relative to the rest of the population $(\sigma)$ on a single fitness peak landscape. The genome length is set to $N=1 \times 10^{4}$. Conservative, semiconservative and semiconservative systems without post-methylation lesion repair are all shown.

in Fig. 4. This turns out to be important, and will be discussed in the next section.

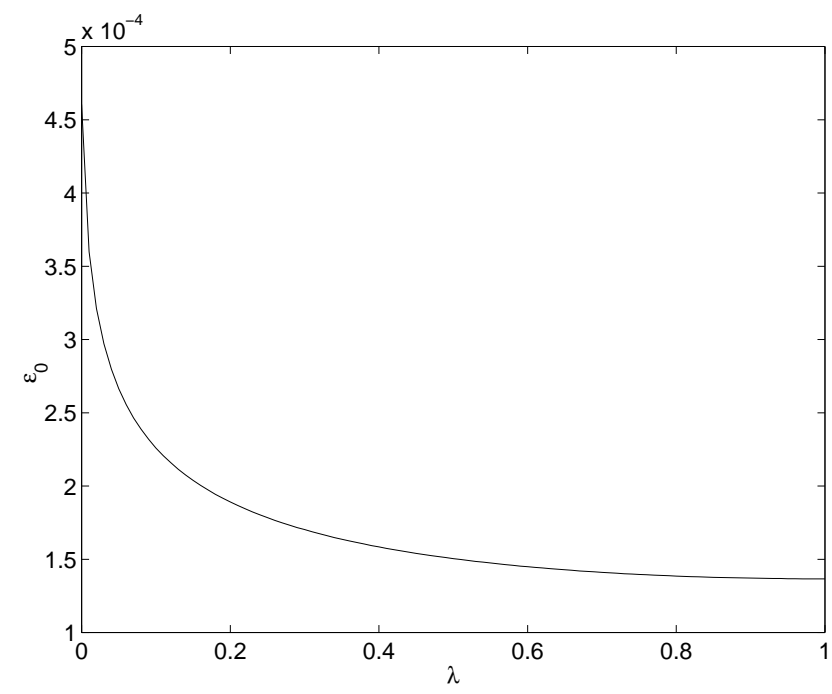

FIG. 4: The error threshold $\left(\epsilon_{0}\right)$ for a semiconservatively replicating population on a single fitness peak landscape vs. the probability of post-methylation DNA repair $(\lambda)$. Here, $N=1 \times 10^{4}$ and $\sigma=1 \times 10^{2}$.

\section{DISCUSSION}

As discussed above, recent results on the semiconservative quasispecies has presented a paradox in tumor 
progression. In the last section, we presented a possible resolution. Although extremely high mutation rates and genetic instability are found in all cancer cells, these mutation rates cannot be handled by a semiconservative genome. However, as lesion repair begins to fail, the error threshold increases. These increased mutation rates may in turn lead to further failure of the lesion repair system and a higher threshold, creating a positive feedback cycle.

The concept of a mutator phenotype in cancer has a long and established history ${ }^{20}$. Failure to prevent and repair mutations is well documented in cancer cells 21 , yet it remains unclear to what extent each repair enzyme is or is not active in any given cell. Thus, it is difficult to say with conviction that lesion repair failure is indeed a prerequisite for the sustenance of genetic instability in MIN genomes in nature. However, there are a number of encouraging signs that this is, in fact, the case. Many human tumors have been found to be deficient in checkpoint pathways, including those that involve p53, p16 and p19ARF ${ }^{20.22}$. These checkpoints are, among other things, designed to increase the efficiency of DNA post-methylation damage repair, and mice that lack these checkpoint genes display abnormally high levels of spontaneous tumor incidence ${ }^{20}$. Loss of the p53 tumor suppression gene has been shown to lead to less efficient GGR $23,24,25$ and mutations of the BRCA1 gene, which enhances the GGR process, and greatly increases the risk of breast cancer in women ${ }^{26}$. Well documented diseases, such as Xeroderma Pigmentosum 21.27.28 are caused by defects in GGR and manifest themselves as an overwhelmingly high probability of tumor development. Expression profiles of pancreatic cancer cells have demonstrated down-regulation of DNA repair genes 29 . All-trans-retinoic acid has been shown to prevent certain carcinogenic transformations by enhancing DNA repair through checkpoint effects ${ }^{30}$. Lastly, numerous human studies have shown positive correlations between individual fluctuations in DNA repair capability and cancer risk $^{31}$.

Although it is clear that DNA repair is linked to tumor suppression, we are suggesting a fundamentally different outlook on the problem. Rather than simply protecting the genome from mutations, DNA repair also prevents the proliferation of genomes with high mutation rates. As methyl-directed mismatch repair begins to fail, the error threshold will soon be crossed, unless DNA repair begins to fail as well. The available experimental evidence shows a definite correlation between repair failure and cancer risk, but causation is not evident. As well, alternative hypotheses can explain this correlation (as repair failure makes genetic instability more likely, and genetic instability makes repair failure more probable), so more evidence is required. One fundamental and novel aspect of our hypothesis, although, of course enormously difficult to practically implement, is that reinstating lesion repair in a full-blown cancer cell should lower the error threshold and provide the same effect as pushing the cells past the error threshold, without the added side effects associated with introducing mutagens into the body.

One must take great care in making grand statements regarding complex biological systems from simplified physics model. Complex processes involving numerous enzymes and prefactors are incorporated into first-order rate constants, and the various types of DNA damage are ignored in favor of simple point mutations. Further, DNA repair covers a complex set of phenomena, rather than the simple post-methylation mismatch repair treated in the model. However, the quasispecies model has been impressively successful in dealing with a wide variety of complex systems, including finite populations ${ }^{32,33.34}$, time dependent landscapes ${ }^{14,15,35}$, punctuated equilibrium 32.36 and even the accurate prediction of human B-cell mutation rates 5 and viral properties 6.7. Despite its simplicity, the model seems to capture the robust properties of genomic evolution. Furthermore, it is successful at all mutation rates, whereas many theories of population genetics only work at low mutation rates, which obviously does not apply to genetically unstable tumor progression. Regarding the fitness landscape, although cancerous genomes can be highly heterogeneous, the single fitness peak landscape likely captures the general features of local behavior even on more complex landscapes, and can be shown to yield the same behavior as more delocalized landscapes. As well, the mathematical isomorphisms shown in the appendices hold for all landscapes and $\mathrm{W}$ matrices.

Lastly, although the model is restricted to errors in the form of point mutations, these are the major source of genetic instability in the MIN (microsatellite instability) tumors, which can be found in $13 \%$ of sporadic colon cancers 37 and all hereditary nonpolyposis colorectal cancer. MIN tumors can display a point mutation rate one thousand times greater than that of a normal cell ${ }^{18,19}$. Other tumors display genetic instability in the form of CIN (chromosomal instability) with a wide variation in chromosome number and other chromosomal instability 19.28. The possibility that such instability can be treated, to a first approximation, through the inclusion of recombination ${ }^{38,39}$ and simulation techniques is the subject of future research ${ }^{40}$. Previous results in these areas provide reason to believe that the underlying dynamics for models of CIN tumors should provide similar results to those obtained here.

\section{CONCLUSIONS}

In this paper, we presented a paradox arising from recent results regarding the quasispecies model of semiconservative replication. The relatively low values for the error threshold, together with the fact that this threshold does not increase with increasing master sequence fitness, suggests that a true semiconservative system should not be capable of handling the exceptionally high mutation rates associated with cancer cells. We demonstrated that, 
through the degradation of lesion repair, the semiconservative system begins to mimic its conservative counterpart, with an increasing error threshold whose upper bound becomes increasingly dependent on the replication rate. Thus, we postulate that the failure of mismatch repair systems and the corresponding increase in mutation rates that are found in MIN tumors must be accompanied by failure of post-methylation lesion repair. Although we present some experimental evidence to support this, the simplicity of the model together with the complexity of the problem require further experimental evidence to fully justify our claim. Thus, we have suggested a new outlook to guide further experimentation and more complex model calculations.

\section{Acknowledgments}

The authors are indebted to Franziska Michor and Emmanuel Tannenbaum for invaluable discussions and a careful reading of the manuscript. This research was supported by an NIH postdoctoral fellowship.

\section{APPENDIX A: SEMICONSERVATIVE QUASISPECIES MODEL WITHOUT POST-METHYLATION DNA REPAIR}

In this appendix we examine a semiconservatively replicating quasispecies model in the absence of any lesion repair. The standard conservative model describes the evolution of a set of organisms, each with a genome $\phi=s_{1} s_{2} \cdots s_{N}$, where each $s_{i}$ represents a "letter" chosen from an alphabet of size $S$. The population fractions obey the set of differential equations ${ }^{3}$

$$
\frac{d x_{\phi}}{d t}=\sum_{\phi^{\prime}} A\left(\phi^{\prime}\right) W\left(\phi, \phi^{\prime}\right) x_{\phi^{\prime}}-f(t) x_{\phi},
$$

where $x_{\phi}$ denotes the fraction of the population with genome $\phi, A(\phi)$ represents the fitness, or growth rate, of sequence $\phi$, and $W\left(\phi, \phi^{\prime}\right)$ is the likelihood of creating sequence $\phi$ from $\phi^{\prime}$ by mutations. $f(t)=\sum_{\phi} A(\phi) x_{\phi}$ is the average fitness of the population, which holds the population size constant and introduces competition. If only point mutations are allowed and a genome-independent mutation probability $\epsilon$ is assumed, then $W\left(\phi, \phi^{\prime}\right)$ can be written in terms of the number of bases at which $\phi$ and $\phi^{\prime}$ differ, the Hamming distance $H D\left(\phi, \phi^{\prime}\right)$, as

$$
W\left(\phi, \phi^{\prime}\right)=\left(\frac{\epsilon}{S-1}\right)^{H D\left(\phi, \phi^{\prime}\right)}(1-\epsilon)^{N-H D\left(\phi, \phi^{\prime}\right)},
$$

where $N$ represents the length of the genome. The isomorphism we are about to describe holds for all $W$, but we shall limit ourselves to this manifestation in appendix $\mathrm{B}$ and $\mathrm{C}$.

For a semiconservative system, organisms are described by a double stranded genome $\left\{\phi, \phi^{\prime}\right\}$, the population fractions as $x_{\left\{\phi, \phi^{\prime}\right\}}$, and the growth rates as $A\left(\phi, \phi^{\prime}\right)$.
It is important to note that, in the absence of lesion repair, $\phi^{\prime}$ is not defined by complementary base pairing to $\phi$, since there is no requirement that base pair mismatches be altered. We use $W\left(\phi, \phi^{\prime}\right)$ as before to describe the probability that replication of the unzipped single stranded genome $\phi^{\prime}$ will produce new strand $\phi$.

Hence, the quasispecies equations for a semiconservative genome without post-methylation DNA repair can be written as

$$
\begin{aligned}
& \frac{d x_{\phi}}{d t}=\sum_{\phi_{a}, \phi_{b}}\left(W\left(\phi, \phi_{a}\right)+W\left(\phi, \phi_{b}\right)\right) A\left(\phi_{a}, \phi_{b}\right) x_{\left\{\phi_{a}, \phi_{b}\right\}}- \\
& \sum_{\phi_{a}} f(t)\left(x_{\left\{\phi, \phi_{a}\right\}}+x_{\left\{\phi_{a}, \phi\right\}}\right),
\end{aligned}
$$

where we define the population fraction $x_{\phi} \equiv$ $\sum_{\phi_{a}} x_{\left\{\phi, \phi_{a}\right\}}+x_{\left\{\phi_{a}, \phi\right\}}$. Note that we count the $5^{\prime} \rightarrow 3^{\prime}$ and $3^{\prime} \rightarrow 5^{\prime}$ strand separately to avoid double counting. Rearranging,

$$
\begin{aligned}
& \frac{d x_{\phi}}{d t}=\sum_{\phi_{a}, \phi_{b}} W\left(\phi, \phi_{a}\right) A\left(\phi_{a}, \phi_{b}\right) x_{\left\{\phi_{a}, \phi_{b}\right\}} \\
& +\sum_{\phi_{a}, \phi_{b}} W\left(\phi, \phi_{b}\right) A\left(\phi_{a}, \phi_{b}\right) x_{\left\{\phi_{a}, \phi_{b}\right\}}-f(t) x_{\phi} \\
& =\sum_{\phi_{a}, \phi_{b}} W\left(\phi, \phi_{a}\right)\left(A\left(\phi_{a}, \phi_{b}\right) x_{\left\{\phi_{a}, \phi_{b}\right\}}+A\left(\phi_{b}, \phi_{a}\right) x_{\left\{\phi_{b}, \phi_{a}\right\}}\right) \\
& -f(t) x_{\phi},
\end{aligned}
$$

where the last expression is obtained by switching the dummy variables $\phi_{a}$ and $\phi_{b}$ in the second summation of Eqn. (A5). We can define an average replication rate for the single strand $\phi$ as

$$
\overline{A(\phi)}=\frac{\sum_{\phi_{a}}\left(A\left(\phi_{a}, \phi\right) x_{\left\{\phi_{a}, \phi\right\}}+A\left(\phi, \phi_{a}\right) x_{\left\{\phi, \phi_{a}\right\}}\right)}{x_{\phi}}
$$

yielding the main result of this appendix,

$$
\frac{d x_{\phi}}{d t}=\sum_{\phi^{\prime}} \overline{A\left(\phi^{\prime}\right)} W\left(\phi, \phi^{\prime}\right) x_{\phi^{\prime}}-f(t) x_{\phi} .
$$

which looks remarkably similar to Eqn. (A1). For large populations, $\overline{A\left(\phi^{\prime}\right)}$ must rapidly equilibrate and remain steady, yielding a system of equations that are identical to that of a conservative quasispecies with a transformed set of rate constants. This is studied in more detail in the particular example of appendix B.

\section{APPENDIX B: SINGLE FITNESS PEAK LANDSCAPE}

In this appendix, we study the system of appendix A evolving on the commonly used single fitness peak 
landscape described below. To solve this problem, we shall explicitly make a number of approximations that have been well studied and found to accurately describe the true dynamics of the system for reasonable genome lengths and population sizes.

The single fitness peak landscape describes the situation where a specific genome perfectly "fits" the environment and hence replicates rapidly, while all other genomes are equally poor replicators. Here, we investigate the case where at least one strand needs to be perfect in order to be viable, a reasonable model for the housekeeping genes responsible for cell survival (other landscapes will be studied in a future work ${ }^{41}$ ). Thus, $A\left(\phi_{a}, \phi_{b}\right)=\sigma \gg 1$ if either $\phi_{a}$ or $\phi_{b}$ are in the set $\left\{\phi_{0}, \phi_{0}^{\prime}\right\}$ which represent the master sequence and its perfect complement, and $A\left(\phi_{a}, \phi_{b}\right)=1$ otherwise.

For large populations and genome lengths, we can ignore mutations from unfit sequences to the master sequence (an approximation that becomes exact as the genome length increases to infinity, but is accurate at realistic finite genome lengths) and assume that, at equilibrium, master genomes are paired with statistically distributed complements (which is exact for this landscape in the large population limit, and rapidly converges for finite populations). Thus, using Eqns. (A6) and (A7) and the fact that the symmetric equations conserve the equality of concentrations of complementary sequences, we can write a differential equation for the sum of the population fractions of the single stranded master genome and its complement, $x_{0}$, and the remaining population, $x_{1}=1-x_{0}$,

$$
\begin{aligned}
& \frac{d x_{0}}{d t}=q^{N} \sigma x_{0}-f(t) x_{0} \\
& \frac{d x_{1}}{d t}=\left(1-q^{N}\right) \sigma x_{0}+\sigma x_{0,1}+x_{1}-x_{0,1} \\
& -f(t) x_{1}
\end{aligned}
$$

where $N$ is the length of the genome, $q$ represents the replicative fidelity, or $1-\epsilon$, where $\epsilon$ is the per base point mutation probability, which is assumed to be sequence independent, $x_{0,1}$ represents the fraction of the population that are imperfect sequences bonded to a perfect sequence (that is, the strands that are not members of $x_{0}$, but are bonded to a member of $x_{0}$ ), and $f(t)=\sigma x_{0}+\sigma x_{0,1}+1-x_{0}-x_{0,1}$. As complements are statistically distributed, we can define $x_{0,1}=F_{0,1} x_{0}$, where $F_{0,1}$ represents the fraction of perfect sequences bonded to imperfect sequences, and is independent of $x_{0}$. We can solve these equations by searching for equilibrium solutions, $\dot{x}_{0}=\dot{x}_{1}=0$, yielding two solutions, the quasispecies solution,

$$
x_{0}=\frac{1-q^{N} \sigma}{\left(1+F_{0,1}\right)(\sigma-1)}
$$

and the quasispecies-free solution, $x_{0}=0$. The error catastrophe occurs when the two solutions meet, i.e., when $x_{0}=0$ in the quasispecies solution. This gives

$$
\sigma=1 / q^{N},
$$

which is identical to the conservative solution and is plotted and discussed in section II.

\section{APPENDIX C: DEGRADATION OF POST-METHYLATION DNA REPAIR}

In this appendix, we evaluate the effect of the progressive failure of post-methylation DNA repair on the single fitness peak landscape discussed in the previous appendix. Setting the probability that an error will be repaired to be $\lambda$, we get

$$
\begin{aligned}
& \frac{d x_{\phi}}{d t}=\sum_{\phi_{a}, \phi_{b}}\left(W\left(\phi, \phi_{a}, \lambda\right)+W\left(\phi, \phi_{b}, \lambda\right)+\right. \\
& \left.W_{2}\left(\phi, \phi_{a}, \lambda\right)+W_{2}\left(\phi, \phi_{b}, \lambda\right)\right) A\left(\phi_{a}, \phi_{b}\right) x_{\left\{\phi_{a}, \phi_{b}\right\}}- \\
& \sum_{\phi_{a}} f(t)\left(x_{\left\{\phi, \phi_{a}\right\}}+x_{\left\{\phi_{a}, \phi\right\}}\right)- \\
& \sum_{\phi_{a}}\left(A\left(\phi, \phi_{a}\right) x_{\left\{\phi, \phi_{a}\right\}}+A\left(\phi_{a}, \phi\right) x_{\left\{\phi_{a}, \phi\right\}}\right),
\end{aligned}
$$

where $W\left(\phi, \phi_{a}, \lambda\right)$ represents the $\lambda$-dependent probability that unzipped strand $\phi_{a}$ will produce new strand $\phi$, while the new quantity $W_{2}\left(\phi, \phi_{a}, \lambda\right)$ represents the probability that, after replication and post-methylation lesion repair of unzipped strand $\phi_{a}$, the erroneous repair of errors will change $\phi_{a}$ to strand $\phi$. A set of manipulations similar to those in appendix A and the definition in Eqn. (A6) can be used to yield the equations

$$
\begin{aligned}
& \frac{d x_{\phi}}{d t}=\sum_{\phi^{\prime}} \overline{A\left(\phi^{\prime}\right)}\left(W\left(\phi, \phi^{\prime}, \lambda\right)+W_{2}\left(\phi, \phi^{\prime}, \lambda\right)\right) x_{\phi^{\prime}}- \\
& (f(t)+\overline{A(\phi)}) x_{\phi}
\end{aligned}
$$

When applied to a single fitness peak landscape, these equations can be written as

$$
\begin{aligned}
& \frac{d x_{0}}{d t}=\left\{\left(1-\frac{\lambda \epsilon}{2}\right)^{N}+\left(1-\left(1-\frac{\lambda}{2}\right) \epsilon\right)^{N}\right\} \sigma x_{0} \\
& -(f(t)+\sigma) x_{0} \\
& x_{1}=1-x_{0}
\end{aligned}
$$

where $x_{0}$ and $x_{1}$ and $f(t)$ are as defined in appendix B. This can be solved for the error threshold, which occurs 
when

$$
\sigma=\frac{1}{(1-\lambda \epsilon / 2)^{N}+(1-(1-\lambda / 2) \epsilon)^{N}-1} .
$$

This expression is plotted and discussed in section II, and approaches the full semiconservative treatment and the solution of appendix B in the limits $\lambda \rightarrow 1$ and $\lambda \rightarrow 0$, respectively.
1 G. M. Cooper, The Cancer Book (Jones and Bartlett Publishing, Boston, MA, 1993).

${ }^{2}$ F. Michor, Y. Iwasa, and M. A. Nowak, Nature Reviews 4, 197 (2004).

3 M. Eigen, J. McCaskill, and P. Schuster, Adv. Chem. Phys. 75, 149 (1989).

4 S. Crotty, C. E. Cameron, and R. Andino, Proc. Nat. Acad. Sci. 98, 6895 (2001).

5 C. Kamp and S. Bornholdt, Phys. Rev. Lett. 88, 068104 (2002).

6 C. Kamp, C. O. Wilke, C. Adami, and S. Bornholdt, Complexity 8, 28 (2003).

7 Y. Brumer and E. I. Shakhnovich, Phys. Rev. E (2004), in press.

8 L. A. Loeb, J. M. Essigmann, F. Kazazi, J. Zhang, K. D. Rose, and J. I. Mullins, Proc. Nat. Acad. Sci. 96, 1492 (1999).

9 R. V. Solé, Eur. Phys. J. B 35, 117 (2003).

10 S. Galluccio, Phys. Rev. E 56, 4526 (1997).

11 P. Schuster and P. F. Stadler, Computers Chem. 18, 295 (1994).

12 P. Tarazona, Phys. Rev. A 45, 6038 (1992).

13 L. Peliti, cond-mat/9712027 (1997).

14 C. O. Wilke, C. Ronnewinkel, and T. Martinetz, Phys. Rep. 349, 395 (2001).

15 M. Nilsson and N. Snoad, Phys. Rev. Lett. 84, 191 (2000).

16 D. Voet and J. Voet, Biochemistry (John Wiley and Sons, Inc., New York, NY, 1995).

17 E. Tannenbaum, E. J. Deeds, and E. I. Shakhnovich, Phys. Rev. E (2004), in press.

18 M. A. Nowak, N. L. Komarova, A. Sengupta, P. V. Jallepalli, L. Shih, B. Vogelstein, and C. Lengauer, Proc. Nat. Acad. Sci. 99, 16226 (2002).

19 D. P. Cahill, K. W. Kinzler, B. Vogelstein, and C. Lengauer, Trends Cell Biol. 9, M57 (1999).

20 L. A. Loeb, K. R. Loeb, and J. P. Anderson, Proc. Nat. Acad. Sci. 100, 776 (2003), and references therein.

21 E. C. Friedberg, G. C. Walker, and W. Siede, DNA Repair and Mutagenesis (ASM Press, Washington, DC, 1995).

22 C. J. Sherr and F. McCormick, Cancer Cell 2, 103 (2002).

23 J. M. Ford and P. C. Hanawalt, Proc. Nat. Acad. Sci. 92, 8876 (1995).

24 J. M. Ford and P. C. Hanawalt, J. Biol. Chem. 272, 28073 (1997).

25 B. J. Hwang, J. M. Ford, P. C. Hanawalt, and G. Chu, Proc. Nat. Acad. Sci. 96, 424 (1999).

26 A. Hartman and J. M. Ford, Nature Genetics 32, 180 (2002).

27 A. S. Balajee and V. A. Bohr, Gene 250, 15 (2000).

28 B. Vogelstein and K. W. Kinzler, The Genetic Basis of Human Cancer (The Mcgraw-Hill Companies, Inc., New York, NY, 1998).

29 T. Crnogorac-Jurcevic, E. Efthimiou, T. Nielsen, J. Loader, B. Terris, G. Stamp, A. Baron, A. Scarpa, and N. R. Lemoine, Oncogene 21, 4587 (2002).

${ }^{30}$ K. H. Dragnev, S. J. Freemantle, M. J. Spinella, and E. Dmitrovsky, Ann. N. Y. Acad. Sci. 952, 13 (2001), and references therein.

31 M. Berwick and P. Vineis, J. Nat. Canc. Inst. 92, 874 (2000), and references therein.

32 Y. Zhang, Phys. Rev. E 55, R3817 (1997).

33 D. Alves and J. F. Fontanari, Phys. Rev. E 57, 7008 (1998).

34 P. R. A. Campos and J. F. Fontanari, Phys. Rev. E 58, 2664 (1998).

35 M. Nilsson and N. Snoad, Phys. Rev. E 65, 031901 (2002).

36 J. Krug and C. Karl, Physica A 318, 137 (2003).

37 K. W. Kinzler and B. Vogelstein, Cell 87, 159 (1996).

38 M. C. Boerlijst, S. Bonhoeffer, and M. A. Nowak, Proc. R. Soc. Lond. B 263, 1577 (1996).

39 L. Barnett, Ph.D. thesis, School of Cognitive and Computing Sciences, University of Sussex (2003).

40 Y. Brumer, F. Michor, and E. I. Shakhnovich (2004), manuscript in preparation.

41 Y. Brumer, E. Tannenbaum, and E. I. Shakhnovich (2004), manuscript in preparation. 